\title{
Molecular Interactions in Monolayers of Azo Dye/Liquid Crystal Mixtures at Interfaces
}

\author{
D. Bauman*, A. PŁóciennik and K. Inglot \\ Faculty of Technical Physics, Poznań University of Technology \\ Nieszawska 13A, 60-965 Poznań, Poland
}

(Received January 15, 2009; in final form June 1, 2009)

\begin{abstract}
A study of azo dye/liquid crystal mixtures in monolayers formed at an air-water interface (the Langmuir films) and at a solid surface (the Langmuir-Blodgett films) has been performed. Five azo dyes with various molecular structure and the liquid crystal 4-octyl-4'-cyanobiphenyl (8CB) have been used. The dyes have been added to the liquid crystal at various molar fractions. Surface pressure and surface potential versus mean molecular area isotherms for the Langmuir films have been recorded and information about intermolecular interactions at the air-water interface has been obtained. On the basis of electronic absorption measurements for the Langmuir and Langmuir-Blodgett films the conclusions about the ability of dyes molecules to form self-aggregates at the interfaces have been drawn. The influence of the dye molecular structure and its concentration on aggregates' geometry has been found.
\end{abstract}

PACS numbers: 68.18.-g, 68.47.Pe, 61.30.Gd, 33.20.-t

\section{Introduction}

Azo dyes provide whole range of colours from which the most popular are red, orange, brown and yellow. Therefore they are one of the most popular group of dyes and have found many applications in industry [1]. Additionally, azo dyes have very high extinction coefficient and give bright, intensive colour by using small concentrations. Because of the large change of the dipole moment at $\pi \rightarrow \pi^{*}$ electronic transition from the ground to the excited state (charge transfer transition), they are characterized by high value of the second order hyperpolarizability [2], thus can find application in nonlinear optics. They are also used as medium in tunable dye lasers and in polarized holography. At high concentrations, azo dyes have tendency to aggregation. The organic materials, in which aggregation phenomena occur, are characterized by different physico-chemical properties in comparison with those of monomeric systems [3]. In particular, they display intriguing optical properties and therefore attract attention as novel functional materials for application in molecular electronics and photonics. Intermolecular interactions leading to the aggregation process can be observed in monomolecular films fabricated by using the Langmuir-Blodgett (LB) technique [4-6]. This technique enables to obtain two-dimensional ordered layers from certain molecules or particles at air- liquid (the Langmuir films) and air-solid substrate (the Langmuir-Blodgett films) interfaces.

Some years ago in our laboratory the study of the molecular organization and aggregation in the Langmuir and Langmuir-Blodgett films formed of azo dyes mixed with a liquid crystal, using as a stabilizing matrix, had been undertaken [7-10]. Our present work, that was carried out as the continuation of the previous study, has aimed at reporting several additional observations related to the intermolecular interactions on the molecular level. In particular, we would like to know the effect of the structure as well as the value and the direction of the dipole moment of dye molecules on their ordering and the tendency to creation of self-aggregates in monolayers at air-water and air-solid substrate interfaces.

\section{Materials and methods}

\subsection{Materials}

Five azo dyes used in this study were synthesized and chromatographically purified in the Institute of Polymer Technology and Dyes at Łódź University of Technology, Poland. The liquid crystal 4-octyl-4'-cyanobiphenyl (8CB) was purchased from the Dabrowski Laboratory at the Military University of Technology, Warsaw (Poland), and was used without further purification. The chemical structure of the compounds used is shown in Table I.

* corresponding author; e-mail: bauman@phys.put.poznan.pl 
TABLE I

The molecular structure of compounds investigated.

Compound

\subsection{Methods}

The Langmuir and Langmuir-Blodgett films were formed in a Minitrough 2 (KSV Instruments Ltd., Finland). This trough was equipped with two barriers for monolayer compression. The subphase was deionized water purified to a final resistivity of $18.2 \mathrm{M} \Omega \mathrm{cm}$ by a Milli-Q system (Millipore Corporation, Austria) and kept at the constant temperature of $(20.5 \pm 0.5)^{\circ} \mathrm{C}$. Monolayers were spread on the water from a chloroform solution of the dye/8CB mixture. An appropriate molar fraction of the dye in the liquid crystal $\left(X_{\mathrm{M}}\right)$ was achieved by dissolving known amounts of both substances in chloroform. After spreading, the solutions were left for $15 \mathrm{~min}$ to ensure complete solvent evaporation before compression was initiated. The monolayer was compressed with the barrier motion speed of $5 \mathrm{~mm} / \mathrm{min}$, while the surface pressure, $\pi$, was monitored by a platinum Wilhelmy plate balance with an accuracy of $\pm 0.1 \mathrm{mN} / \mathrm{m}$. Simultaneously, the surface potential of the monolayer, $\Delta V$, as a function of the mean molecular area $(\Delta V-A$ isotherm) was measured using the vibrating plate method by means of a SPOT 1 head from KSV. An accuracy of this method was $\pm 10 \mathrm{mV}$. All measurements were repeated on fresh subphases three to five times to confirm reproducibility. Standard trough cleaning procedure was adopted between measurements. The monomolecular films were deposited on both sides of polished quartz plates $\left(35 \times 10 \times 1 \mathrm{~mm}^{3}\right)$ with hydrophilic surface for LB film fabrication. The vertical dipping method was used and dipping rate was $5 \mathrm{~mm} / \mathrm{min}$. The dipping stroke was $25 \mathrm{~mm}$. Successful deposition took place only on the first up-stroke of the substrate. The transfer ratio was near unity.
The absorption spectra of chloroform solutions as well as of the Langmuir and LB films were recorded in the visible spectral region by means of a spectrophotometer CARY 400. In order to record absorption spectra of monolayers spread on the water surface in situ, the spectrophotometer was equipped with a fiber optic accessory supplied by Varian. The details of the equipment are described in Ref. [11]. The monolayer was compressed to a predetermined surface pressure and maintained there for 5 min before starting spectral measurements. The absorbance of the surface of pure water was taken as the reference.

\section{Results and discussion}

\subsection{Surface pressure and surface potential as a function of mean molecular area}

The measurement of the surface pressure-mean molecular area $(\pi-A)$ isotherm is a basic and widely used technique to characterize a Langmuir film. The surface pressure $\pi$ is a measure of the change in surface tension of the water covered with a monolayer with respect to the pure water. The $\pi-A$ isotherm represents the plot of the change of the $\pi$ value as a function of the average area available for one molecule on the water surface during compression process. After spreading chloroform solutions of the azo dyes at the water surface in the trough, we found that the dyes produce monolayers which were unable to offer any significant resistance to barriers approach during compression: the $\pi$ value was continuously zero, the molecules were expelled and the bulk phase was observed to form. This means that none of the azo dyes under investigation cannot produce stable and compressible monolayer, although all they possess terminal and/or lateral polar group. Similar behaviour was noticed for azo dyes investigated previously [7-10]. However, when the dyes were mixed with $8 \mathrm{CB}$ at appropriate concentrations, the compression was possible and we obtained $\pi-A$ isotherms. They were recorded for the dye/8CB mixtures up to the molar fraction of the dye equal to 0.5. It was found that for such mixtures just after spreading, microcrystallites in the chloroform solution were not visible with the naked eye. We recorded also $\pi-A$ isotherm during expansion process and for the mixtures up to $X_{\mathrm{M}}=0.5$ only small hysteresis between the both isotherm runs was found. This means that the equilibrium conditions were kept.

Figure 1 presents $\pi-A$ isotherms of the Langmuir films of $8 \mathrm{CB}$ and of $\mathbf{3} / 8 \mathrm{CB}$ mixtures, as examples, whereas the characteristic values of $\pi-A$ isotherms for monolayers of all mixtures investigated are resumed in Table II. The following data are presented: $A_{0}^{\pi}$ - the value of the area at which the surface pressure begins to rise (the first edge of the phase transition), $A_{\mathrm{C}}$ - the value of the collapse area, and $\pi_{\mathrm{C}}$ - the value of the collapse pressure (see inset in Fig. 1). The collapse point is recognized as the point in the isotherm where the derivative $\partial \pi / \partial A$ begins to decrease. The values of $A_{\mathrm{C}}$, which are greater than the 
molecular cross-section $\left(\approx 0.17 \mathrm{~nm}^{2}\right.$ for $8 \mathrm{CB}$ molecule), suggest that in the monolayers the rigid cores of the dye and liquid crystal molecules must be tilted with respect to the water surface. Assuming that the mean molecular area in the Langmuir film is determined by the rigid core of the molecule and taking the experimental $A$ value into account, it is possible to calculate the tilt angle. The tilt angle $\varphi$ for $8 \mathrm{CB}$ was estimated as the angle between the long axis of the rigid core of liquid crystal molecules and the normal to the water surface just before the collapse point. It is in agreement with the data given in literature [12-14]. From the course of the $\pi-A$ isotherms for the dye/8CB mixtures it follows that the rise of $\pi$ begins at smaller and smaller values as $X_{\mathrm{M}}$ of the dye increases. This indicates that the presence of the dye improves the packing of molecules in the Langmuir film. As a result the tilt angle decreases. Knowing the area of the dyes molecules and taking into account the mixture composition, we were able to estimate the average angles $\varphi$ for mixed monolayers. They are gathered in Table II, too. One should keep, however, in the mind that such estimation is very rough, because in general the splay deformation of the Langmuir film can occur.

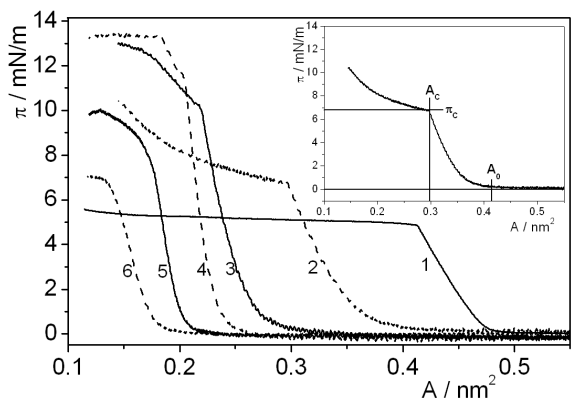

Fig. 1. Surface pressure-mean molecular area $(\pi-A)$ isotherms of Langmuir films of pure 8CB (1) and of binary mixtures of dye 3 in $8 \mathrm{CB}$ with $X_{\mathrm{M}}$ of 0.1 (2), $0.2(3), 0.3(4), 0.4(5)$ and $0.5(6)$.

The increase of the dye content in the mixture causes the better rigidity and stability of the Langmuir film, as is confirmed by the rise of the isotherm steepness and the value of $\pi_{\mathrm{C}}$. The characteristic dye concentration is $X_{\mathrm{M}}=0.3$. At such content of the dye, both the tilt angle of the $\pi-A$ plot (with respect to the horizontal direction) and $\pi_{\mathrm{C}}$ have the highest values. That means that any azo dye molecules are able to "stiffen" the molecules of $8 \mathrm{CB}$. As the molar fraction of the dye increases further, $\pi_{\mathrm{C}}$ value decreases indicating the diminishing of the monolayer stability. Although the compression is still possible, in the most cases the average area per molecule is too small for even the most dense packing of molecules in a monolayer, which suggests the creation of three-dimensional (3D) domains.

The changes of the isotherm run with the Langmuir film composition for the dye/8CB mixtures indicate on the interactions between dye and liquid crystal molecules.
TABLE II

Features of $\pi-A$ and $\Delta V-A$ isotherms, the average angle between the long molecular axis and the normal to the water surface, $\varphi$, and the effective dipole moment, $\mu_{\perp}$, for the Langmuir films of azo dye/8CB mixtures.

\begin{tabular}{|c|c|c|c|c|c|c|c|c|}
\hline Comp. & $X_{\mathrm{M}}$ & $\begin{array}{c}A_{0}^{\pi} \\
{\left[\mathrm{nm}^{2}\right]}\end{array}$ & $\begin{array}{c}A_{\mathrm{C}} \\
{\left[\mathrm{nm}^{2}\right]}\end{array}$ & $\begin{array}{c}\pi_{\mathrm{C}} \\
{\left[\mathrm{mN} \mathrm{m}^{-1}\right]}\end{array}$ & $\begin{array}{c}\varphi \\
{\left[{ }^{\circ}\right]}\end{array}$ & $\begin{array}{l}A_{0}^{\Delta V} \\
{\left[\mathrm{~nm}^{2}\right]}\end{array}$ & $\begin{array}{c}\Delta V_{\mathrm{C}} \\
{[\mathrm{V}]}\end{array}$ & $\begin{array}{l}\mu_{\perp} \\
{[\mathrm{D}]}\end{array}$ \\
\hline $8 \mathrm{CB}$ & - & 0.48 & 0.41 & 4.8 & 61 & 0.65 & 630 & 0.68 \\
\hline \multirow[t]{5}{*}{$\mathbf{1} / 8 \mathrm{CB}$} & 0.1 & 0.42 & 0.32 & 5.4 & 31 & 0.65 & 618 & 0.61 \\
\hline & 0.2 & 0.35 & 0.28 & 6.1 & 21 & - & - & - \\
\hline & 0.3 & 0.32 & 0.24 & 7.0 & 15 & 0.70 & 524 & 0.40 \\
\hline & 0.4 & 0.25 & 0.21 & 4.8 & 12 & - & - & - \\
\hline & 0.5 & 0.26 & 0.20 & 4.0 & 11 & 0.67 & 352 & 0.23 \\
\hline \multirow[t]{5}{*}{$2 / 8 \mathrm{CB}$} & 0.1 & 0.46 & 0.37 & 5.2 & 35 & 0.60 & 624 & 0.61 \\
\hline & 0.2 & 0.32 & 0.26 & 5.8 & 20 & - & - & - \\
\hline & 0.3 & 0.30 & 0.24 & 7.0 & 15 & 0.45 & 461 & 0.29 \\
\hline & 0.4 & 0.27 & 0.22 & 11.6 & 13 & - & - & - \\
\hline & 0.5 & 0.25 & 0.20 & 9.0 & 10 & 0.50 & 388 & 0.23 \\
\hline \multirow[t]{5}{*}{$3 / 8 \mathrm{CB}$} & 0.1 & 0.43 & 0.29 & 6.7 & 33 & 0.52 & 607 & 0.52 \\
\hline & 0.2 & 0.32 & 0.22 & 10.2 & 22 & - & - & - \\
\hline & 0.3 & 0.27 & 0.20 & 11.6 & 19 & 0.50 & 411 & 0.27 \\
\hline & 0.4 & 0.22 & 0.17 & 7.8 & 14 & - & - & - \\
\hline & 0.5 & 0.20 & 0.14 & 6.7 & 11 & 0.37 & 465 & 0.23 \\
\hline \multirow[t]{5}{*}{$4 / 8 \mathrm{CB}$} & 0.1 & 0.47 & 0.37 & 4.9 & 45 & 0.60 & 640 & 0.70 \\
\hline & 0.2 & 0.43 & 0.34 & 5.0 & 38 & - & - & - \\
\hline & 0.3 & 0.39 & 0.30 & 5.1 & 30 & 0.47 & 671 & 0.57 \\
\hline & 0.4 & 0.37 & 0.29 & 4.4 & 27 & - & - & - \\
\hline & 0.5 & 0.27 & 0.20 & 4.2 & 17 & 0.44 & 627 & 0.40 \\
\hline \multirow[t]{5}{*}{$\mathbf{5} / 8 \mathrm{CB}$} & 0.1 & 0.44 & 0.37 & 4.6 & 45 & 0.53 & 636 & 0.64 \\
\hline & 0.2 & 0.43 & 0.36 & 4.3 & 40 & - & - & - \\
\hline & 0.3 & 0.37 & 0.31 & 4.3 & 31 & 0.45 & 638 & 0.54 \\
\hline & 0.4 & 0.33 & 0.26 & 4.2 & 24 & - & - & - \\
\hline & 0.5 & 0.27 & 0.23 & 4.1 & 19 & 0.38 & 600 & 0.37 \\
\hline
\end{tabular}

The kind of these interactions can be followed in Fig. 2, in which the excess of the average area per molecule, $A_{\mathrm{E}}=A_{12}-\left(X_{\mathrm{M} 1} A_{1}+X_{\mathrm{M} 2} A_{2}\right)$, as a function of $X_{\mathrm{M}}$ for the dye/8CB mixtures in the Langmuir films is presented. $A_{12}$ is here the average molecular area in the two-component film, $X_{\mathrm{M} 1}$ and $X_{\mathrm{M} 2}$ are the mole fractions of the components, and $A_{1}$ and $A_{2}$ are the single component areas at the same $\pi$. The values of $A$ were taken at $\pi=4 \mathrm{mN} / \mathrm{m}$. The negative deviation from the additivity rule, which is distinctly observed for dyes $\mathbf{1}, \mathbf{2}$ and 3 mixed with $8 \mathrm{CB}$, means a contraction of the two-component films due to attractive interactions between molecules of both components $[15,16]$. The positive values of $A_{\mathrm{E}}$ indicate on repulsive intermolecular interactions $[15,16]$ and they occur here in $\mathbf{4} / 8 \mathrm{CB}$ and $\mathbf{5} / 8 \mathrm{CB}$ mixtures. The values of $A_{\mathrm{E}} \neq 0$ for all the binary mixtures investigated would suggest that two components in the Langmuir films are always good miscible. However, the information from the surface phase rule [15] should be additionally taken into account. This rule states that if the components are miscible, the $\pi_{\mathrm{C}}$ value should change with the mixture composition. Thus, the dependence of the $\pi_{\mathrm{C}}$ value on the composition of the Langmuir films of 
the dye/liquid crystal mixtures seen in Table II indicates that in the case of $4 / 8 \mathrm{CB}$ and $5 / 8 \mathrm{CB}$ mixtures the immiscibility or at least only partial miscibility of the dye and liquid crystal molecules can be postulated. This results from the fact that although the additivity rule is not fulfilled, $\pi_{\mathrm{C}}$ remains constant.

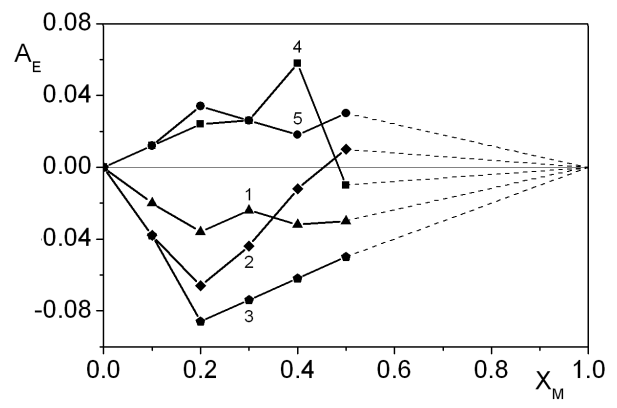

Fig. 2. Plot of the excess of the mean molecular area per molecule, $A_{\mathrm{E}}$ of dyes $\mathbf{1}(1), \mathbf{2}(2), \mathbf{3}$ (3), 4 (4), and 5 (5) mixed with $8 \mathrm{CB}$ versus $X_{\mathrm{M}}$ of a dye at $\pi=$ $4 \mathrm{mN} / \mathrm{m}$.

Additional information about molecular organization in the Langmuir film, particularly at early stages of the film compression when the surface pressure is still zero, can be gained from the surface potential-area isotherm. The surface potential can be related to an average effective dipole moment for the monolayer-forming molecules by Helmholtz equation $[17,18]$ :

$$
\Delta V=\mu_{\perp} / A \varepsilon_{\mathrm{r}} \varepsilon_{0},
$$

where $\mu_{\perp}=\mu \cos \gamma$ is the average vertical component of the molecular dipole moment, $\gamma$ is the angle between the surface normal and the dipole moment direction (in general, it has not to be along the long molecular axis), $A$ is the mean area per molecule, and $\varepsilon_{\mathrm{r}}$ and $\varepsilon_{0}$ are the dielectric constant of the monolayer and the electric permittivity of the free space, respectively.

Figure 3a presents, reproduced from Ref. [9], the electric surface potential-area isotherm (solid curve), recorded simultaneously with the $\pi-A$ isotherm (dotted curve), as well as the effective dipole moment-area isotherm (dashed curve) for the liquid crystal 8CB, which can be treated as a reference for the mixtures of azo dyes with 8CB. Exemplary results for the Langmuir films of $\mathbf{2} / 8 \mathrm{CB}$ and $\mathbf{5} / 8 \mathrm{CB}$ mixtures at $X_{\mathrm{M}}=0.3$ are shown in Figs. $3 b$ and $c$. In all the cases, the surface potential starts rising earlier than the surface pressure. $\Delta V$ first increases rather sharply up to the area which coincides with the area of the $\pi$ rise onset. Next $\Delta V$ keeps on growing but at a slower rate and reaches the maximum at the collapse point. The values of the area at which the surface potential starts to rise, $A_{0}^{\Delta V}$, the surface potential at the collapse point, $\Delta V_{\mathrm{C}}$, and the effective dipole moment $\mu_{\perp}$ calculated from Eq. (1) taking $A=A_{\mathrm{C}}$ for the mixtures under investigation are presented in Table II. Behind the collapse point $\Delta V$ remains constant or drops down very slightly. As the surface potential is contributed only by the monolayer coming into contact with the water [19], this suggests that during the compression, the molecules do not assume more and more vertical alignment. They have to be "pushed out" above the first monolayer being in contact with the water. Therefore, the dipole moment $\mu_{\perp}$ calculated from Eq. (1) for 8CB and its mixtures with the azo dyes has no physical meaning beyond the collapse point and is not shown in Figs. $3 \mathrm{a}-\mathrm{c}$.

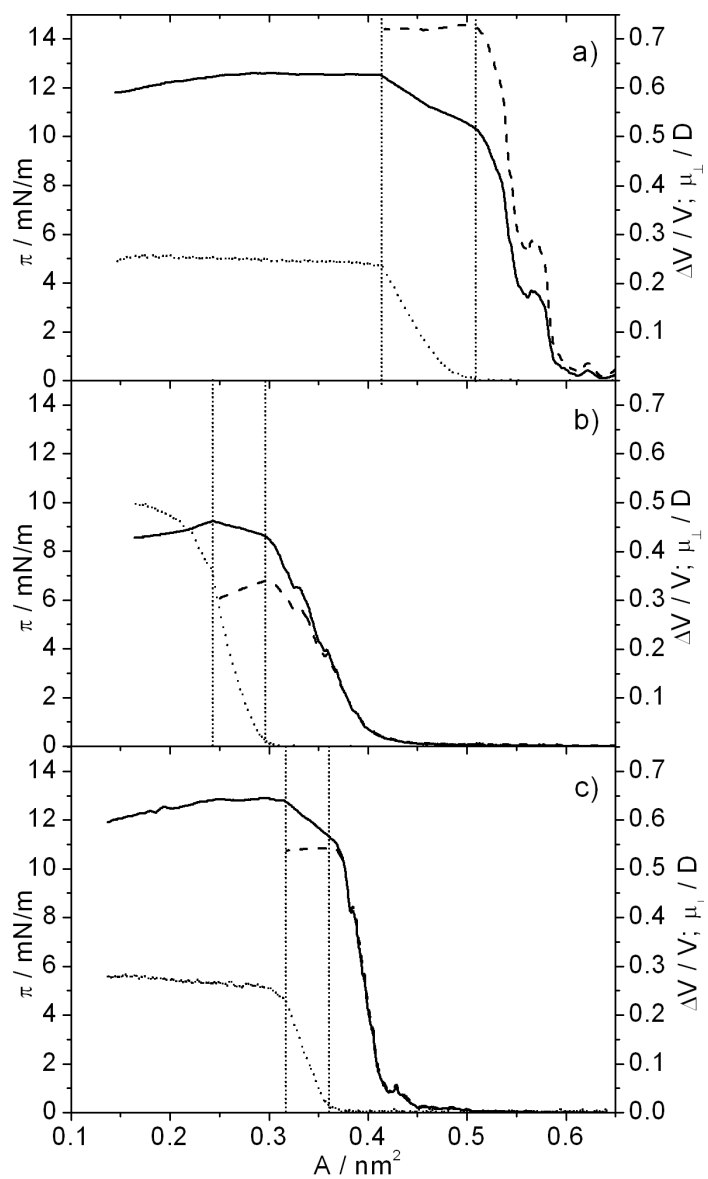

Fig. 3. Surface potential, $\Delta V$ (solid curve), surface pressure, $\pi$ (dotted curve), and effective dipole moment, $\mu_{\perp}$ (dashed curve) as a function of the mean molecular area, $A$, for Langmuir film of $8 \mathrm{CB}$ (a) and $2 / 8 \mathrm{CB}(\mathrm{b})$, $5 / 8 \mathrm{CB}$ (c) mixtures at $X_{\mathrm{M}}=0.3$ (the scale in ordinate axis for $\Delta V$ and $\mu_{\perp}$ is the same).

The quantity $\mu_{\perp}$ is so-called apparent dipole moment and cannot be mistaken for the dipole moment of an isolated molecule, because it is influenced by several dipole fields. According to the Demchak-Fort model [20], $\mu_{\perp}$ is composed of three independent contributions arising from (i) orientation of water dipoles in the vicinity of the headgroups, (ii) the headgroup dipoles, and (iii) the hydrophobic part dipoles. Moreover, in our calculations we assumed, as it is made customary $[19,21]$, that $\varepsilon_{\mathrm{r}}=1$, although it is known that dipoles in a monolayer may be embedded in media with distinct, nonunity dielectric constant values, which depends on whether these 
dipoles are at the monolayer-water or monolayer-air interface $[22,23]$.

For $8 \mathrm{CB}$ the rapid increase of $\mu_{\perp}$ together with the rise of $\Delta V$ is observed, up to the highest value of $0.68 \mathrm{D}$ being reached at the area corresponding to the onset for the surface pressure. Such a value of $\mu_{\perp}$ remains constant up to the collapse point (the product $\Delta V \cdot A$ is constant in the region between vertical lines in Figs. $3 \mathrm{a}-\mathrm{c}$ ). Similar behaviour is observed for the dye/liquid crystal mixtures, solely for some mixtures small diminishing of $\mu_{\perp}$ is observed (see for example Fig. $3 \mathrm{~b}$ ). The runs of $\mu_{\perp}-A$ isotherms indicate that at the large mean molecular area, the liquid crystal and dye molecules lie almost horizontally at the air-water interface $\left(\mu_{\perp}=0\right)$. Upon compression, the molecules start to interact and at some critical area $\left(A_{0}^{\Delta V}\right)$ the hydrophobic parts of the liquid crystal molecules lift up from the water surface resulting in their tilted alignment with respect to the horizontal direction. Such changes of the molecules orientation are confirmed by the significant increase of $\Delta V$ and $\mu_{\perp}$ at this stage. Since $\mu_{\perp}$ reaches the maximal value at the area where the surface pressure starts to increase and then remains constant up to the collapse point, we suppose that the molecular orientation does not change in this region. This means that the tilt angle of the dye and liquid crystal molecules at the air-water interface $(\varphi)$ is settled already at the beginning of the formation of the condensed monolayer.

From the data presented in Table II it follows that with the increase of the dye content in the mixture, although $\pi_{\mathrm{C}}$ values rises or remains constant, $\Delta V_{\mathrm{C}}$ decreases. As a result, $\mu_{\perp}$ calculated from Eq. (1) for the dye/liquid crystal mixtures is always smaller than that for the pure liquid crystal and decreases when the $X_{\mathrm{M}}$ of the dye rises. This is rather unexpected result taking into account the fact that the molecules in the Langmuir films formed of the mixtures align more and more perpendicularly to the water surface with the increase of the dye content (see $\varphi$ values in Table II). The same effect was observed for monolayers of other azo dyes mixed with liquid crystals $[9,24]$ and was explained in terms of the opposite orientation of the dye dipole moments with respect to the dipole moments of the liquid crystal molecules. Such antiparallel alignment of the dipole moments leads to the diminishing of the resultant $\mu_{\perp}$ value.

Similarly as was made in Ref. [9], we looked for the correlation between the value and the direction of the dipole moment of the given dye molecule and the diminishing of $\mu_{\perp}$ values of the monolayer after the dye addition. Dyes $\mathbf{1}, \mathbf{3}$ and $\mathbf{4}$ have similar value of the dipole moment, with different directions of it. The value of the dipole moment of dyes $\mathbf{2}$ and $\mathbf{5}$ is smaller because here the lateral $-\mathrm{NO}_{2}$ group $(\mu \approx 4 \mathrm{D})$ is replaced by $-\mathrm{Cl}$ group $(\mu \approx 1.5 \mathrm{D})$. However, the values of $\mu_{\perp}$ calculated from Eq. (1) are not associated with these facts. Meanwhile, the smallest changes of $\mu_{\perp}$ appear for 4/8CB and 5/8CB mixtures. As in these mixtures the immiscibility or at least partial miscibility of the dye and liquid crystal molecules is noticed, it means that the dipole-dipole interaction between both kinds of molecules is hindered making the creation of the antiparallel pairs consisting of the dye and liquid crystal molecules more difficult. The results obtained point out on the strong correlation between the value of the total effective dipole moment and the miscibility of components in the monolayer formed of azo dye/8CB mixtures at the air-water interface. The model of the mutual alignment of the dipole moments of the liquid crystal and azo dye molecules in the case of the good miscibility and the phase separation of both components in the binary mixtures was proposed by us in Ref. [9]. The results obtained here are very good confirmation of this model.

\subsection{Electronic absorption spectra of Langmuir and Langmuir-Blodgett films}

The measurements of the electronic absorption were carried out both for the Langmuir and LangmuirBlodgett films formed of all prepared azo dye/liquid crystal mixtures. Figure 4 shows the UV-Vis absorption spectra of the LB films of $1 / 8 \mathrm{CB}$ mixtures at various $X_{\mathrm{M}}$, as examples. In the spectra two main absorption bands can be distinguished. The band in UV region with the maximum at $\approx 280 \mathrm{~nm}$ is related to the absorption of $8 \mathrm{CB}$ with very small contribution of the dye absorption. However, the band in the visible region is assigned to the $\pi \rightarrow \pi^{*}$ electronic transition in the dye molecule with the transition moment parallel to its long axis. Table III gathers the maximum position, $\lambda_{\max }$, and half-bandwidth, $\delta$, for azo dyes mixed with $8 \mathrm{CB}$ in the Langmuir and the LB films and, for comparison, dissolved in chloroform at a molar fraction of $1.5 \times 10^{-7}$. The solution spectra are characteristic for azo dyes in the monomeric form. The absorption spectra of azo dye/8CB mixtures in the films are strongly broadened and, except of dye $\mathbf{3}$ in the LB films, shifted towards longer wavelengths with respect to the spectra in chloroform. This observation could suggest that in the monolayers at the air-water and air-solid substrate interfaces some fraction of self-aggregates by the dye molecules is created.

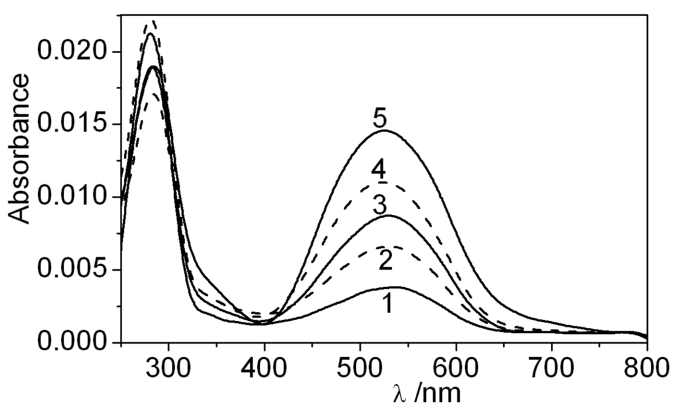

Fig. 4. Absorption spectra of LB films formed of 1/8CB mixtures with $X_{\mathrm{M}}$ of a dye of 0.1 (1), 0.2 (2), 0.3 (3), 0.4 (4), and 0.5 (5). 
TABLE III

The position of absorption maximum, $\lambda_{\max }$, and the half-bandwidth, $\delta$, of the long-wavelength absorption band of azo dyes dissolved in chloroform and azo dye/8CB mixtures embedded in the Langmuir and LB films. For $\lambda_{\max }, \Delta \lambda= \pm 1 \mathrm{~nm}$, for $\delta, \Delta \delta=$ $\pm 10 \mathrm{~cm}^{-1}$ for chloroform and $\pm 50 \mathrm{~cm}^{-1}$ for $8 \mathrm{CB}$.

\begin{tabular}{|c|c|c|c|c|c|c|c|}
\hline \multirow{3}{*}{ Dye } & \multirow{2}{*}{\multicolumn{2}{|c|}{$\begin{array}{c}\text { Chloroform } \\
X_{\mathrm{M}}=1.5 \times 10^{-7}\end{array}$}} & \multicolumn{5}{|c|}{$8 \mathrm{CB}$} \\
\hline & & & \multirow[b]{2}{*}{$X_{\mathrm{M}}$} & \multicolumn{2}{|c|}{ Langmuir film } & \multicolumn{2}{|c|}{ LB film } \\
\hline & $\begin{array}{l}\lambda_{\max } \\
{[\mathrm{nm}]}\end{array}$ & $\begin{array}{c}\delta \\
{\left[\mathrm{cm}^{-1}\right]}\end{array}$ & & $\begin{array}{l}\lambda_{\max } \\
{[\mathrm{nm}]}\end{array}$ & $\begin{array}{c}\delta \\
{\left[\mathrm{cm}^{-1}\right]}\end{array}$ & $\begin{array}{l}\lambda_{\max } \\
{[\mathrm{nm}]}\end{array}$ & $\begin{array}{c}\delta \\
{\left[\mathrm{cm}^{-1}\right]}\end{array}$ \\
\hline \multirow[t]{5}{*}{1} & 499 & 4700 & 0.1 & 534 & 5050 & 537 & 5100 \\
\hline & & & 0.2 & 530 & 5100 & 530 & 5200 \\
\hline & & & 0.3 & 524 & 5250 & 529 & 5150 \\
\hline & & & 0.4 & 524 & 5250 & 524 & 5500 \\
\hline & & & 0.5 & 525 & 5450 & 524 & 5550 \\
\hline \multirow[t]{5}{*}{2} & 481 & 4800 & 0.1 & 520 & 5000 & 518 & 4950 \\
\hline & & & 0.2 & 512 & 4950 & 507 & 5300 \\
\hline & & & 0.3 & 510 & 5100 & 504 & 5400 \\
\hline & & & 0.4 & 506 & 5250 & 508 & 5400 \\
\hline & & & 0.5 & 505 & 5350 & 508 & 5450 \\
\hline \multirow[t]{5}{*}{3} & 536 & 3900 & 0.1 & 560 & 4500 & 520 & 6800 \\
\hline & & & 0.2 & 566 & 4650 & 514 & 7100 \\
\hline & & & 0.3 & 571 & 4650 & 522 & 7250 \\
\hline & & & 0.4 & 565 & 5000 & 522 & 7300 \\
\hline & & & 0.5 & 552 & 5900 & 524 & 7350 \\
\hline \multirow[t]{5}{*}{4} & 527 & 3650 & 0.1 & 535 & 3800 & 531 & 3900 \\
\hline & & & 0.2 & 535 & 3900 & 531 & 4150 \\
\hline & & & 0.3 & 530 & 4100 & 531 & 4350 \\
\hline & & & 0.4 & 538 & 4450 & 544 & 4550 \\
\hline & & & 0.5 & 546 & 4500 & 556 & 4650 \\
\hline \multirow[t]{5}{*}{5} & 512 & 3600 & 0.1 & 520 & 3900 & 521 & 3950 \\
\hline & & & 0.2 & 522 & 3950 & 529 & 4000 \\
\hline & & & 0.3 & 530 & 4000 & 534 & 4100 \\
\hline & & & 0.4 & 535 & 4000 & 534 & 4100 \\
\hline & & & 0.5 & 539 & 4250 & 541 & 4100 \\
\hline
\end{tabular}

The molar extinction coefficient of azo dyes is sufficiently large (over 20000) in order to expect at such concentrations as in the Langmuir and LB films the dipole dipole interaction leading to the exciton coupling. On the basis of the molecular exciton model $[25,26]$, it is known that the interaction between transition dipoles results in the splitting of the energy level of the excited state into two levels with higher and lower energy relative to the undisturbed excited state (the Davydov splitting).

Assuming the parallel configuration of dyes 1-5 molecules in the simplest aggregate, i.e. in the dimer, the co-planar arrangement of the absorption transition moments can be considered. In this case the transition to one of the excited states, corresponding to an antiparallel alignment of dipole moments, is forbidden, while the energy difference between the excited monomer state and the exciton state depends on the angle $\Theta$ between the dipole transition moment of the molecule and the vector joining the centers of two dipoles as follows [26]:

$$
\Delta E_{\mathrm{NK}}=\frac{|\boldsymbol{M}|^{2}}{\boldsymbol{R}^{3}}\left(1-3 \cos ^{2} \Theta\right) .
$$

$\boldsymbol{M}$ is the electric dipole transition moment of the molecule, lying along the long axis, $\boldsymbol{R}$ is the center to center distance vector between two molecules in dimer, and $\Theta$ is the angle between $\boldsymbol{M}$ and $\boldsymbol{R}$.

When the alignment of the dipole moments in the aggregates is such that $0^{\circ}<\Theta<54.7^{\circ}$, the exciton band is located energetically below the monomer band causing a bathochromic shift of the absorption spectrum, and created aggregates are called J-aggregates [27]. For $54.7^{\circ}<\Theta<90^{\circ}$, the exciton band is located energetically above the monomer band causing a hypsochromic shift, and corresponding aggregates are referred to as H-aggregates [27]. When $\Theta=54.7^{\circ}$, no shift in the absorption spectrum is observed, and the aggregates are then called I-aggregates [28].

Looking at the data presented in Table III it is seen that the absorption band of all the dyes in the monolayers becomes broader and broader as the dye content increases. However, the shift of the maximum position is different for various dyes. For the azo dyes studied previously, it was found $[8,10]$ that at low concentrations of a dye in the Langmuir and LB films the absorption maximum position shifts towards longer wavelengths with respect to the position of the monomeric absorption band. Upon increasing the content of the dye, the wavelength of the maximum band shortens. Thus, we had suggested that at the change of the dye concentration in the films various kinds of aggregates are formed. The detailed analysis of the absorption spectra had revealed that the mutual orientation of molecules in the aggregate as well as the amount of the appropriate kind of aggregates at given $X_{\mathrm{M}}$ strongly depend on the molecular structure of the dye, and first of all on the value and the direction of the resultant dipole moment. However, for all the mixtures investigated the general observation had been made that at a low dye content apart from monomers, only J-dimers appear. When the dye concentration rises, the $\mathrm{H}$-dimers can be also created.

We tried to separate the long-wavelength absorption spectra for the dye/8CB mixtures under investigation into two or three bands, corresponding to the absorption of monomers, J- and/or H-dimers. As a model function for experimentally obtained absorption band of the dye/liquid crystal mixtures in the Langmuir and LB films we used a sum of normalized Gaussians. We found that dyes $\mathbf{1}$ and $\mathbf{2}$ behave similarly as the dyes from Refs. [8, 10]: at a low dye content in the mixture the monomers and some fraction of J-type dimers occur, whereas at $X_{\mathrm{M}}=0.5$ the monomers, J-dimers and $\mathrm{H}$-dimers in various fractions exist. In the case of dyes $\mathbf{4}$ and $\mathbf{5}$ the creation of J-dimers was observed only. With the rise of the dye content more and more aggregates appear and the amount of the monomers decreases, however H-type dimers are not created. It seems that the direction of the dipole moment in the molecules of dyes $\mathbf{4}$ and $\mathbf{5}$ is not conducive to such alignment of the 
molecules that enables H-dimers creation. The fractions of the monomers and the appropriate dimers in the mixtures of dyes $\mathbf{1}, \mathbf{2}, \mathbf{4}$, and $\mathbf{5}$ with $8 \mathrm{CB}$ at given concentrations are similar in the Langmuir and LB films. The shapes of the absorption band are almost the same, solely the absorbance of the monolayer on the water surface is lower than that of the monolayer on the quartz surface, especially at higher dye concentrations. This is illustrated in Fig. 5a for $1 / 8 \mathrm{CB}$ mixture at $X_{\mathrm{M}}=0.5$, as an example. As the absorption intensity is related to the projection of the absorption transition moment on the water or quartz slide surface, the smaller absorbance of the Langmuir film means that the average angle between the long molecular axis and the normal to the surface is smaller than in the LB film. This indicates that at the transfer of the monolayer from the water surface to the quartz plate, a rearrangement of the molecular organization takes place. Such effect was previously observed for other compounds $[8,10,11,29-32]$. The rearrangement of the molecules alignment during the transfer was found also for dye $\mathbf{3}$, but quite different than in the case of other dyes. The absorption spectra of $\mathbf{3} / 8 \mathrm{CB}$ mixture in the Langmuir and LB films at $X_{\mathrm{M}}=0.5$ are presented in Fig. 5b, and Fig. 6 shows the best fitting for the decomposition of these spectra into Gaussian-type absorption bands. The molecules of dye $\mathbf{3}$ create $\mathrm{H}$-type dimers in the LB film, which appear already at $X_{\mathrm{M}}=0.1$. As the dye content rises, small fraction of J-dimers in the mixture is presented, too (Fig. 6a). In contrary, in the Langmuir film, at a low dye content, apart of the monomers, J-type dimers exist. When $X_{\mathrm{M}}$ increases, the monomers join together forming both J-type and H-type aggregates (Fig. 6b). The large fraction of J-dimers causes that the absorbance of the Langmuir film is greater than that of the LB film. This results from the fact that, as follows from the measurements of the absorption by using polarized light [8], molecules in J-type aggregates are significantly more tilted with respect to the substrate surface than molecules in H-type aggregates.

\section{Conclusions}

Any of the azo dyes under investigation cannot produce the compressible monolayer at the air-water interface. However, when the dyes are mixed with $8 \mathrm{CB}$ it is possible to obtain compressible Langmuir films on the water surface up to the molar fraction of $X_{\mathrm{M}}=0.5$. The analysis of $\pi-A$ isotherms of the Langmuir films revealed that properties of the monolayer on the water surface (packing density, stability, and rigidity) depend strongly on the structure of the dye molecules as well as on the mixture composition. The surface potential measurements pointed out that in the spread monolayer the molecules of the dye are aligned with the dipole moment directed mostly opposite to the dipole moment of the liquid crystal molecules, i.e. from the water to the air. The influence of the miscibility or the immiscibility of both components in the Langmuir film on the value of the resultant

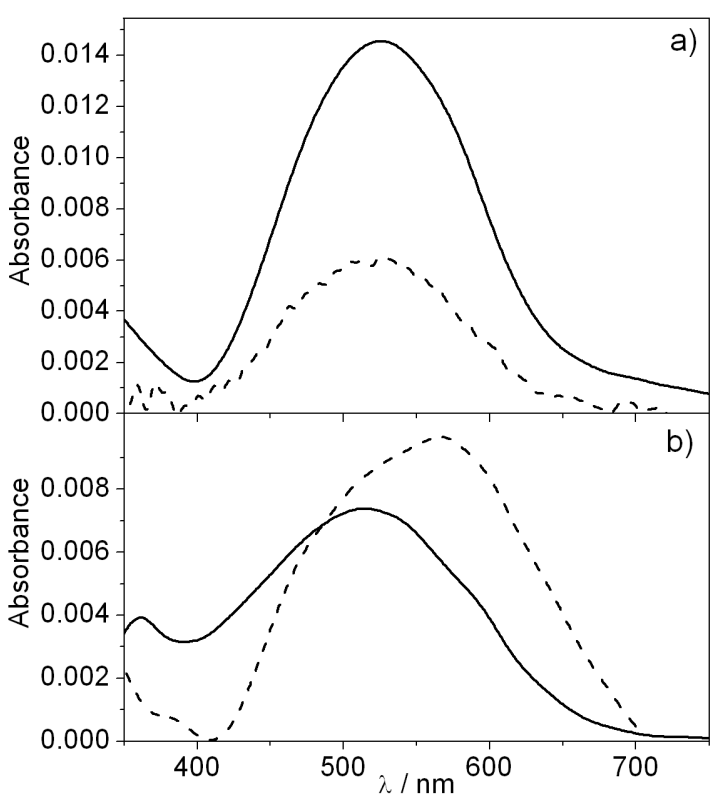

Fig. 5. Long-wavelength absorption spectra of LB (solid curve) and Langmuir (dashed curve) films of 1/8CB (a) and 3/8CB (b) mixtures at $X_{\mathrm{M}}=0.5$.

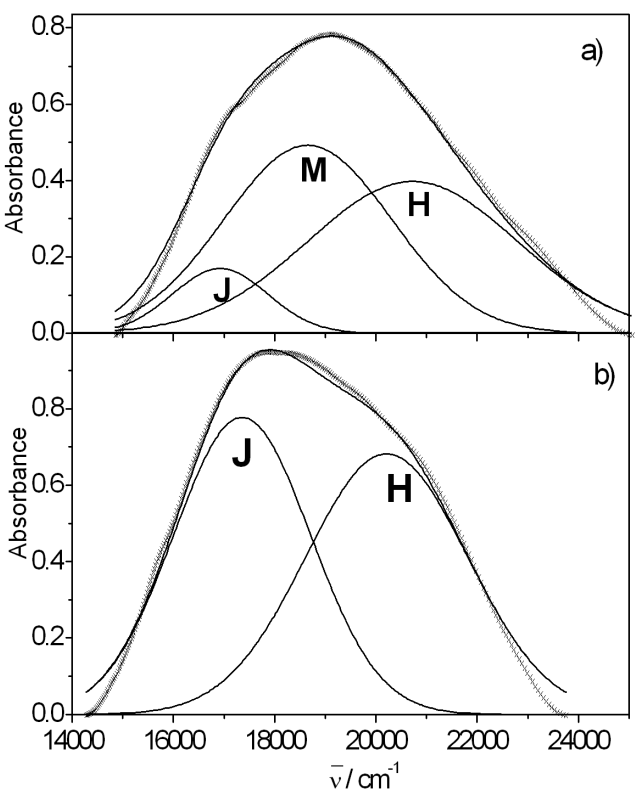

Fig. 6. Decomposition of the absorption spectrum of LB (a) and Langmuir (b) films of $3 / 8 \mathrm{CB}$ mixture at $X_{\mathrm{M}}=0.5$ into Gaussian-type absorption bands.

effective dipole moment was found. The electronic absorption spectra recorded for dye/8CB mixtures in both the Langmuir and LB films showed very modest changes with respect to the spectra of the dyes in the diluted solution, primarily in the form of a broadening of the spectrum. The shift of the maximum position of the absorption band, different for various dye and dependent on 
the dye molar fraction, was also observed. It was found that in the monolayers at interfaces J-type and H-type aggregates are created by azo dye molecules. The fraction and the type of aggregates strongly depend on the molecular structure of the dye and can be quite different in both kinds of the monolayers. Moreover, during the transfer of the monolayer from the water surface onto the quartz substrate the rearrangement of the molecular organization took often place.

In final conclusion it should be emphasized that the ability of azo dyes to aggregation in LB films indicates that such systems can be considered as promising products to use in molecular electronics and photonics devices as well as in nonlinear optics. The dyes have to be, however, first embedded in a stabilizing matrix, e.g. in one of thermotropic liquid crystal.

\section{Acknowledgments}

This work was supported by Poznań University of Technology Research Project No. 64-001/2009-DS.

\section{References}

[1] H. Meier, in: The Chemistry of Synthetic Dyes, Ed. K. Venkataraman, Vol. IV, Academic Press, New York 1971, p. 389.

[2] Side-Chain Liquid Crystal Polymers, Ed. C.B. Mc Ardle, Blackie, Glasgow 1984.

[3] Organic Molecular Aggregates: Electronic Excitation and Interaction Processes, Eds. P. Reineker, H. Haken, H.C. Wold, Springer, Berlin 1983.

[4] G. Roberts, Langmuir-Blodgett Films, Plenum Press, New York 1990

[5] A. Ulman, An Introduction to Ultrathin Organic Films: from Langmuir-Blodgett to Self-Assembly, Academic Press, New York 1991.

[6] M.C. Petty, Langmuir-Blodgett films - An Introduction, Cambridge University Press, Cambridge 1996.

[7] T. Martyński, A. Biadasz, D. Bauman, Liq. Cryst. 29, 281 (2002).

[8] K. Inglot, A. Kaleta, T. Martyński, D. Bauman, Dyes Pigments 77, 303 (2008).

[9] K. Inglot, T. Martyński, D. Bauman, Dyes Pigments 80, 106 (2009).

[10] K. Inglot, T. Martyński, D. Bauman, Opto-Electron. Rev. 17, 120 (2009).
[11] A. Biadasz, K. Łabuszewska, E. Chrzumnicka, E. Michałowski, T. Martyński, D. Bauman, Dyes Pigments 74, 598 (2007).

[12] J. Xue, C.S. Jung, M.W. Kim, Phys. Rev. Lett. 69, 474 (1992).

[13] K. Inglot, T. Martyński, D. Bauman, Liq. Cryst. 33 , 855 (2006).

[14] A. Biadasz, T. Martyński, R. Stolarski, D. Bauman, Liq. Cryst. 31, 1639 (2004).

[15] G.L. Gaines, Insoluble Monolayers at Liquid-Gas Interface, Interscience, New York 1966.

[16] A. Angelova, M. van der Auweraer, R. Ionov, D. Vollhardt, F.C. De Schryver, Langmuir 11, 3167 (1995).

[17] J.T. Davies, E.K. Rideal, Interfacial Phenomena, Academic Press, New York 1963.

[18] D. Myers, Surfaces, Interfaces and Colloids, WileyVCH, New York 1999.

[19] P. Schmitz, H. Gruler, Europhys Lett. 29, 451 (1995).

[20] R.J. Demchak, T.J. Fort, Jr., J. Colloid Interface Sci. 46, 191 (1974).

[21] P. Dynarowicz-Łątka, Adv. Colloid Interface Sci. 45, 215 (1993).

[22] O.N. Oliveira, Jr., D.M. Taylor, T.J. Lewis, S. Salvagno, C.J.M. Stirling, J. Chem. Soc. Faraday Trans. 85, 85 (1989).

[23] D.M. Taylor, G.F. Baynes, Mater. Sci. Eng. C 8-9, 65 (1999).

[24] T. Martyński, A. Biadasz, D. Bauman, Z. Naturforsch. 58a, 97 (2003).

[25] A.S. Davydov, Theory of Molecular Excitons, Mc Graw-Hill Book Co., New York 1962.

[26] M. Kasha, H.R. Rawls, M. Ashraf El-Bayoumi, Pure Appl. Chem. 11, 371 (1965).

[27] D. Moebius, Adv. Mater. 7, 437 (1995).

[28] A. Miyata, D. Heard, Y. Unuma, Y. Higashigaki, Thin Solid Films 210/211, 175 (1992).

[29] T. Kawai, J. Umemura, T. Takenaka, Langmuir 5, 1378 (1989).

[30] E. Johnson, R. Aroca, Y. Nagao, J. Phys. Chem. 95 8840 (1991).

[31] R. Hertmanowski, T. Martyński, D. Bauman, J. Mol. Struct. 741, 201 (2005).

[32] A. Biadasz, T. Martyński, R. Stolarski, D. Bauman, Liq. Cryst. 33, 307 (2006). 